\title{
Neural Development of Speech Sensorimotor Learning
}

\author{
Hiroki Ohashi ${ }^{1,2}$ and David J. Ostry ${ }^{1,2}$ \\ ${ }^{1}$ Department of Psychology, McGill University, Montréal, Québec H3A 1G1, Canada, and ${ }^{2}$ Haskins Laboratories, New Haven, Connecticut 06511
}

The development of the human brain continues through to early adulthood. It has been suggested that cortical plasticity during this protracted period of development shapes circuits in associative transmodal regions of the brain. Here we considered how cortical plasticity during development might contribute to the coordinated brain activity required for speech motor learning. Specifically, we examined patterns of brain functional connectivity (FC), whose strength covaried with the capacity for speech audio-motor adaptation in children ages 5-12 and in young adults of both sexes. Children and adults showed distinct patterns of the encoding of learning in the brain. Adult performance was associated with connectivity in transmodal regions that integrate auditory and somatosensory information, whereas children rely on basic somatosensory and motor circuits. A progressive reliance on transmodal regions is consistent with human cortical development and suggests that human speech motor adaptation abilities are built on cortical remodeling, which is observable in late childhood and is stabilized in adults.

Key words: speech sensorimotor learning; brain development; multisensory integration; resting-state fMRI

\section{Significance Statement}

A protracted period of neuro plasticity during human development is associated with extensive reorganization of associative cortex. We examined how the relationship between FC and speech motor learning capacity are reconfigured in conjunction with this cortical reorganization. Young adults and children aged 5-12 years showed distinctly different patterns. Mature brain networks related to learning included associative cortex, which integrates auditory and somatosensory feedback in speech, whereas the immature networks in children included motor regions of the brain. These patterns are consistent with the cortical reorganization that is initiated in late childhood. The result provides insights into the human biology of speech as well as to the mature neural mechanisms for multisensory integration in motor learning.

\section{Introduction}

Nonhuman primates are endowed with vocal tracts that are capable of generating sounds like human speech (Fitch et al., 2016), but even chimpanzees that were raised from birth by humans are unable to learn to produce speech sounds (Kellogg, 1968). This stands in contrast to the human ability to learn speech sensorimotor control so well that human adults are able to adjust articulatory movements to acquire a novel sensorymotor association in a matter of minutes (Houde and Jordan, 1998; Tremblay et al., 2003). The human ability for speech learning demands coordination of movements of various articulators in sequence and simultaneous monitoring of auditory and

\footnotetext{
Received Nov. 13, 2020; revised Mar. 11, 2021; accepted Mar. 15, 2021.

Author contributions: H.O. and D.J.O. designed research; H.O. performed research; H.O. contributed unpublished reagents/analytic tools; H.O. analyzed data; and H.O. and D.J.O. wrote the paper.

This study was supported by National Institute on Deafness and Other Communication Disorders Grants R01DC012502 and R01DC017439 to D.J.0. We thank Eric Holgate, Angus Campbell, Jacqueline Turcios, Ricardo Ruy Valle-Mena, and Quintisha Upchurch for assistance in the data collection; Eric Holgate, Mark Tiede, and Takayuki Ito for assistance in the experimental setup; and Ananda Sidarta for comments on the analysis and on the paper.

The authors declare no competing financial interests.

Correspondence should be addressed to David J. 0stry at david.ostry@mcgill.ca.

https://doi.org/10.1523/JNEUROSCI.2884-20.2021

Copyright $\odot 2021$ the authors
}

somatosensory feedback to achieve speech sensory goals. Because of these complex demands of speech learning, the neural circuits subserving speech learning in young adults are composed of diverse associative regions of the brain including prefrontal, temporal, and parietal cortex as well as primary sensory and motor cortices (Tourville et al., 2008; Golfinopoulos et al., 2011; Niziolek and Guenther, 2013; Zheng et al., 2013; Darainy et al., 2019; Johnson et al., 2019; Floegel et al., 2020). Plastic changes associated with speech motor learning occur in a network spanning these associative regions (Floegel et al., 2020). A remaining question is how coordinated brain activity across diverse regions of the brain emerges over the course of development.

A key to this question may lie in the protracted schedule of human neurobiological development. The primary beneficiary of this extended window of plasticity in the human brain is associative cortex: synaptic densities in human primary visual and somatosensory cortex rapidly drop during childhood while synaptic pruning in the prefrontal cortex continues through to early adulthood (Sherwood and Gómez-Robles, 2017). These changes in associative cortex contribute to a reorganization during this period of functional and structural connectivity (Sotiras et al., 2017; Baum et al., 2020; Váša et al., 2020). To date, the behavioral focus on this work has been on cognitive function (Baum et al., 
2020). However, given the contributions of associative cortex to speech motor control, the neural circuits involved in speech learning may emerge as part of this substantial reorganization of brain functional connectivity (FC). Nevertheless, there is conflicting evidence with regard to this possibility for speech development. Specifically, studies of speech motor learning have found no behavioral changes in speech audio-motor adaptation with development after 4 years of age (Shiller et al., 2010; MacDonald et al., 2012; Daliri et al., 2018; Caudrelier et al., 2019; Kim et al., 2020; van Brenk and Terband, 2020). Consistent with these behavioral findings, the pattern of brain activity in simple speech production is similar for children and adults as we will show in the present study, although adults' activity is greater in temporal and parietal cortex (Krishnan et al., 2015). This suggests that the basic architecture of the speech learning circuit may mature in childhood, and subsequent changes in the circuit may be relatively minor.

To distinguish these possibilities, we examined how strengths of resting-state functional connectivity were aligned with the capacity for speech learning in children ages 5-12 and also in young adults. The capacity for speech learning was assessed using adaptation to altered auditory information, which is referred to as altered auditory feedback, an experimental model of speech motor learning. Comparisons between the two age groups revealed distinct patterns in the relationship between brain activity and learning. Connectivity strength, which varied with learning, was observed in associative regions of the brain in adults, whereas learning-related connectivity was observed in sensorimotor regions in children. This result is consistent with the possibility that the cortical circuit for speech learning emerges as part of a cortical reorganization that targets associative regions of the brain.

\section{Materials and Methods}

Experimental design and statistical analysis. Twenty-four adults (9 males and 15 females; age 18-30years) and 19 children (8 males and 11 females; age 5-12 years; Fig. $1 A$ for age distribution), all monolingual speakers of English participated in this study. All subjects were right-handed and had no prior neurologic or speech disorders. They had not participated previously in studies involving speech audio-motor adaptation. The Human Investigation Committee of Yale University approved the experimental protocol. Adult subjects provided written informed consent, and child subjects provided assent with parental informed consent.

The experiment was designed to identify the neural substrates of the behavioral plasticity observed in audio-motor adaptation in speech production at different stages of human development. The subjects each participated in a magnetic resonance imaging (MRI) session followed by a behavioral session (Fig. 1B). The MRI session consisted of a structural image acquisition, a speech localizer scan, and resting-state scans. In the behavioral session, subjects produced the task word "beb" (/b $\epsilon \mathrm{b} /)$ while receiving altered auditory feedback that resulted in the signal sounding more like "bab" (/bæb/).

Statistical analyses were conducted within each of the adult and child groups and between the two groups. Details of the analysis for behavioral data can be found below in Behavioral data analysis. Details of the analysis for imaging data are given below in Functional connectivity analysis and Psychophysiological interaction analysis.

Imaging data acquisition. Functional connectivity is known to reflect motor and perceptual processes as well as individual traits and thus can be a probe to identify the brain basis of variability in human behaviors and development. To associate functional connectivity with learning performance, intrinsic brain activity was measured before speech audiomotor adaptation.

The MRI session was conducted in a Siemens Tim Trio 3 tesla MRI scanner with a 32-channel head coil at the Yale Magnetic Resonance
A
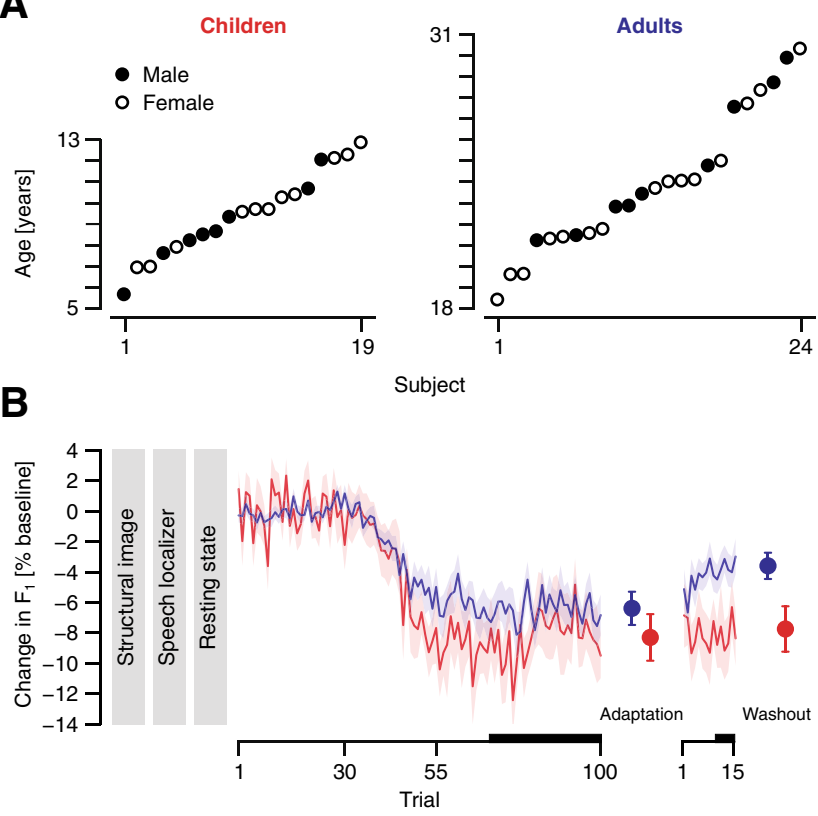

Figure 1. Profiles of subjects and experimental design. $\boldsymbol{A}$, Age and gender distributions of child and adult subjects. $\boldsymbol{B}$, The experimental design and behavioral performance of adults (blue) and children (red) in the speech audio-motor adaptation task. Circles and error bars represent mean values and SEs of changes in the first formant frequency over each of last 30 trials at the hold phase and the last five trials of the washout phase. Shaded areas represent SES.

Research Center. The session consisted of a structural image acquisition, a gradient field map acquisition, and functional image acquisitions in a speech-localizer scan and in two resting-state scans. The structural image was acquired with a T1-weighted 3D magnetization-prepared rapid gradient-echo sequence (repetition time, $\mathrm{TR}=2530 \mathrm{~ms}$; echo time, $\mathrm{TE}=2.77 \mathrm{~ms}$; slices $=256$; thickness $=1.0 \mathrm{~mm}$ isotropic with no gap). The functional images were acquired with a multiband $2 \mathrm{D}$ echo-planar imaging sequence $(\mathrm{TR}=1300 \mathrm{~ms}$; $\mathrm{TE}=48.2 \mathrm{~ms}$; slices $=72$; thickness $=2.0 \mathrm{~mm}$ isotropic with no gap; multiband acceleration factor $=6$ ). Subjects laid supine on a scanner bed wearing insert earphones with their heads held in place with foam pads.

The speech-localizer scan was used to define regions of interest (ROIs) for a resting-state functional connectivity analysis. 226 volumes were acquired in a $294 \mathrm{~s} \mathrm{scan}$. Subjects were instructed to listen to words and to repeat them aloud one time each. They were asked to say their name when they heard a word they could not identify. Twenty-nine words were presented through the insert earphones in a rapid eventrelated design with jittered interstimulus intervals. The schedule of the word presentations was constant across all subjects.

In the resting-state scans, subjects were instructed to lie quietly with their eyes closed. Two 226-volume recordings were obtained with a 294 s scan for each.

Speech audio-motor adaptation. Altered auditory feedback (AAF; Houde and Jordan, 1998) was used to measure speech audio-motor adaptation. In this experimental paradigm, subjects are instructed to produce a task word that typically includes a specific vowel. The vowel sounds produced by subjects are altered to sound similar to another vowel and are played back to subjects through headphones in real time. Vowels are acoustically characterized by peaks in the envelope of the sound spectrum, called formant frequencies, and can be altered to sound like another vowel by shifting the formant frequencies. An upward shift of the lowest formant [first formant frequency $\left(\mathrm{F}_{1}\right)$ ] makes $/ \epsilon /$ sound similar to /æ/ (" $\mathrm{e}$ " to "a"), whereas a downward shift makes / $\epsilon$ / sound similar to /I/ ("e" to "i"). When such an alteration is experienced, subjects adaptively change their pronunciation to compensate for the acoustical error regardless of whether they are aware of it or not (Munhall et al., 2009).

In the present study, subjects wore headphones and a head-mounted microphone and sat in front of a monitor in a soundproof room. They 
were instructed to produce the task word "beb" when a cartoon character appeared on the monitor. Speech sounds were recorded through a head-mounted microphone and digitally sampled at $44.1 \mathrm{kHz}$. In parallel to the recording, an acoustical effects processor (VoiceOne, TC Helicon) altered $\mathrm{F}_{1}$ of the recorded sound in real time by shifting up the formant frequency of the sounds lower than $1.5 \mathrm{kHz}$, without changing the pitch (Rochet-Capellan and Ostry, 2011; Shiller and Rochon, 2014). The altered sounds were mixed with pink noise to prevent subjects from hearing their own voice via air and bone conduction and then played back to subjects through headphones. The sound volume of speech was adjusted on a per-subject basis before starting the speech session. The ratio of the amplitude of pink noise to that of acoustical feedback was held constant across subjects in this study.

Subjects produced the task word in total 115 times. The initial 30 trials were in a baseline phase in which auditory feedback was not altered. The baseline $F_{1}$ value in adults and children was $686 \pm 18.6$ (SE) and $735 \pm 19.6 \mathrm{~Hz}$, respectively. $F_{1}$ was gradually shifted upward over the next 25 trials (the ramp phase), and then the maximal shift was maintained for the following 45 trials (the hold phase). The resultant shifts in percentage terms in $F_{1}$ in the hold phase averaged $23.9 \pm 1.59$ (SE) and $26.0 \pm 2.73 \%$ for adults and children, respectively. There was no significant difference in the proportionate change in formant frequency between these two groups $\left(t_{(29.6)}=0.700, p=0.489, d=0.226\right.$; Welch's $t$ test). The feedback alteration was turned off for the last 15 trials (the washout phase). There was one child who did not complete the washout phase. Subjects were instructed to speak as usual and to keep the sound volume constant.

Behavioral data analysis. The dependent measure for the speech behavioral session was the amount of audio-motor adaptation and that of the washout. The speech acoustical signal was resampled at $16 \mathrm{kHz} . \mathrm{F}_{1}$ and second formant frequency $\left(\mathrm{F}_{2}\right)$ were estimated from the vowel sounds of the resampled data using Praat software (Boersma and Weenink, 2018). The vowel sounds were detected based on the intensity and harmonics-to-noise ratio of the speech sounds. The lowest five peaks of the spectral envelope were estimated from the vowel sounds every $5 \mathrm{~ms}$ with a $25 \mathrm{~ms}$ Hamming window using linear predictive coding (LPC) implemented with Burg's algorithm (Anderson, 1978). The LPC order was selected on a per-subject basis to minimize total SDs of $F_{1}$ and $\mathrm{F}_{2}$. Formant frequencies were tracked based on the time series of the five peaks using the Viterbi algorithm. Representative $F_{1}$ values for each trial were obtained by taking the mean value over $30 \mathrm{~ms}$ centered on the vowel sound. Individual trials in which $F_{1}$ values were beyond 2 SDs from the mean were excluded in subsequent analyses. The time course of $F_{1}$ over the session was normalized as the proportionate change relative to the mean $F_{1}$ over all trials in the baseline phase (trials 1-30). Onesample $t$ tests with Bonferroni-Holm correction for the number of subjects $(N=43)$ were applied to the normalized $\mathrm{F}_{1}$ values to test whether individual subjects adapted to altered auditory feedback (one-tailed corrected $p<0.01$ ). The amount of adaptation was quantified as the mean normalized $F_{1}$ over last 30 trials in the hold phase (trials 71-100), and this value was used in subsequent functional connectivity analyses (see below, Functional connectivity analysis). The amount of washout was assessed as the difference between the amount of adaptation and the mean normalized $\mathrm{F}_{1}$ over the last five trials in the washout phase (trials 111-115)

Group-level analyses were conducted to test changes in speech production over a course of the speech adaptation session and differences in the $\mathrm{F}_{1}$ changes between adults and children. Specifically, the amount of adaptation and washout (percentage changes relative to baseline) were tested against zero for each of the adults and children using one-sample $t$ tests. Each of these two measures was also compared between adults and children using Welch's $t$ tests. A series of the $t$ tests was followed by Bonferroni-Holm correction for multiple comparisons. The proportion of children versus adults who were found to have adapted was compared using a $\chi^{2}$ test. Effect sizes were computed with Cohen's $d$ and Cohen's $h$.

Subjects' concentration on the speech task may account for betweensubject differences in the measure of adaptation. For example, a particular child who was less concentrated on the task may show less adaptation to AAF. To address this issue, we assessed the reaction time (RT) that was taken to produce the task word after the initiation of the trial. RTs were averaged over all trials on a per-subject basis, and then the relationship between RT and the amount of adaptation was tested in each of adults and children using Spearman partial correlation controlling for the age of the subjects.

Imaging data preprocessing. The brain extraction from the structural images was performed using optiBET (Lutkenhoff et al., 2014) and used for the registration of functional images into standard space. The skullstripped image was also segmented into cortical and subcortical regions using Freesurfer (version 5.3.0; Fischl, 2012) to identify white matter and ventricle regions. The identified regions were used in the resting-state data analysis to remove nuisance signals.

Functional images acquired in the speech-localizer scan and the two resting-state scans were preprocessed using AFNI (versions 19.2.16 and 19.2.26; Cox, 1996), except for the static magnetic field (B0) correction and the independent component analysis (ICA), which were both conducted using FSL (version 5.0.9; Jenkinson et al., 2012). The processing consisted of the removal of first two volumes, B0 correction using fieldmap images, slice-timing correction, motion correction, alignment between the functional and structural images, nonlinear registration onto the ICBM 2009c nonlinear asymmetric template in Talairach space, provided by AFNI, and spatial smoothing with full-width-at-half-maximum (FWHM) $5 \mathrm{~mm}$. For the resting-state data, after the B0 correction, spike events were identified in the preprocessed data and replaced to fit a smooth curve.

Accuracy of the nonlinear registration. The imaging data of children as well as adults were registered onto the adult brain template, ICBM 2009c, to enable statistical comparisons of brain activity in the common space. The mismatch between the template image of adults and the morphology of the brain of children might cause registration errors and thereby contaminate statistical comparisons between adults and children. To assess this possibility, we evaluated the difference in accuracy of the registration conducted for adults and children. Planes representing the central sulcus, lateral sulcus, superior temporal sulcus, and inferior precentral sulcus of the individual brains and of the ICBM 2009c brain were created by manually tracing the sulci on each slice of the brain image with $3 \mathrm{~mm}$ lines. These sulci were selected because these were easily traceable and could be anatomic landmarks for our ROIs. The planes representing the sulci of individual brains were aligned using the nonlinear registration that was used in the imaging data preprocessing and then compared with the ICMB 2009c planes using the Dice coefficient. This process quantified the overlap of the sulci of individual brains and those of the ICBM 2009c brain. If the accuracy of the registration for children is worse than that for adults, then Dice coefficients for children should be lower than those for adults. This hypothesis was tested by Welch's $t$ test on arcsine-transformed Dice coefficients.

ROI identification. In an individual-level analysis following preprocessing, the processed image was scaled and then a general linear model (GLM) analysis was conducted using AFNI's 3dREMLfit. GLM was performed including regressors for experimental design convolved with AFNI's SPMG2 basis function (canonical hemodynamic response function with its temporal derivative), the 12 motion parameters (mean and temporal derivative of the motion parameters), and a polynomial function accounting for a trend signal, controlling autocorrelation structure of the signals. Adjacent TRs in which motion between successive time points exceeded $0.35 \mathrm{~mm}$ were censored out. $2.49 \pm 0.924$ (SE) and $27.9 \pm 4.46 \%$ of volumes were censored out in adults and children, respectively. The proportion of volumes censored out in adults was significantly smaller than that in children $\left(t_{(19.6)}=-5.58, p<0.0001, d=\right.$ -1.91 ; Welch's $t$ test).

The group-level analysis identified brain regions showing positive and negative blood-oxygen-level-dependent (BOLD) responses to the speech task using a mixed-effects model implemented using AFNI's 3dMEMA. The smoothness of the image was modeled as a nonGaussian spatial autocorrelation function (ACF), averaged at a group level and used in AFNI's 3dClustSim to obtain nearest-neighbor, facetouching, two-sided cluster thresholds via a Monte Carlo simulation (Cox et al., 2017; two-tailed voxel-wise $p<0.002$, cluster significant level 
Table 1. List of ROls used in the resting-state analysis

\begin{tabular}{|c|c|c|c|c|c|c|c|c|}
\hline \multirow[b]{2}{*}{ ROI } & \multicolumn{4}{|c|}{ Right hemisphere (mm) } & \multicolumn{4}{|c|}{ Left hemisphere (mm) } \\
\hline & $\mathrm{RL}$ & $\mathrm{AP}$ & IS & $Z$ & $\mathrm{RL}$ & AP & IS & $z$ \\
\hline S1/M1 & -48 & 10 & 33 & 9.85 & 48 & 8 & 32 & 9.24 \\
\hline $\mathrm{A} 1$ & -43 & 19 & 8 & 8.53 & 36 & 28 & 10 & 9.42 \\
\hline Pre-SMA & & & & & 2 & 1 & 60 & 8.21 \\
\hline IFG & -56 & -12 & 11 & 3.94 & 52 & -12 & 11 & 5.91 \\
\hline OP1 & -36 & 23 & 15 & 7.86 & 39 & 22 & 14 & 8.00 \\
\hline $\mathrm{PF}$ & -54 & 26 & 22 & 5.01 & 53 & 40 & 24 & 7.91 \\
\hline aSTG/STS & -57 & 1 & -5 & 8.58 & 51 & 5 & -2 & 6.76 \\
\hline $\mathrm{pSTG/STS}$ & -62 & 23 & 7 & 9.42 & 60 & 24 & 9 & 8.85 \\
\hline $\mathrm{Pu}$ & -20 & -1 & 8 & 8.52 & 19 & -1 & 8 & 8.93 \\
\hline CbVI & -19 & 55 & -22 & 9.09 & 25 & 55 & -22 & 9.81 \\
\hline CbVIII & -28 & 45 & -46 & 5.41 & 32 & 40 & -42 & 4.67 \\
\hline
\end{tabular}

Coordinates are given in Talairach space in RAI order. $z_{1} z$-Values of activity in the localizer session computed from data taken from adults and children together; RL, right-left; AP, anterior-posterior; IS, inferior-superior.

$\alpha<0.01$ ). The group-level analysis was conducted for each of withinadults, within-children, and across-groups to define ROIs for each.

ROIs for the seed-based functional connectivity analysis (see below, Functional connectivity analysis) were selected based on the statistical result obtained in the across-groups analysis. ROIs were defined as 6 $\mathrm{mm}$ spheres centered on the local maxima in the speech-localizer task in the following target areas: primary somatosensory and motor cortex (S1/ M1), primary auditory cortex (A1), secondary somatosensory cortex (OP1), presupplementary motor area (pre-SMA), posterior superior temporal gyrus and sulcus (pSTG/STS), anterior superior temporal gyrus and sulcus (aSTG/STS), anterior supramarginal gyrus (PF), inferior frontal gyrus (IFG), putamen $(\mathrm{Pu})$ and cerebellum lobule VI (CbVI) and VIII (CbVIII). Table 1 shows lists of ROIs and associated Talairach coordinates [in mm, Right-Anterior-Inferior (RAI) order]. Peak activity was located at the central sulcus, between M1 and S1. Accordingly, we did not select ROIs for these regions separately.

The resting-state data preprocessing. ICA, implemented using FSL's MELODIC (Beckmann and Smith, 2004), followed the common preprocessing (see above, Imaging data preprocessing) to obtain nuisance signals to be regressed out. Specifically, after removing a polynomial function accounting for a trend signal from the preprocessed data, independent components (ICs) were estimated from the detrended data by MELODIC and manually labeled as noise or not in accordance with guidelines recommended in previous studies (Kelly et al., 2010; Griffanti et al., 2017). Furthermore, to keep criteria for the noise classification constant across images, FMRIB's ICA-based Xnoiseifier (FIX; Griffanti et al., 2014) was trained based on our own hand-labeling and the spatiotemporal features of ICs of all images, and then the trained FIX classified the ICs as noise or not. The number of ICs automatically determined by MELODIC was on average $47.0 \pm 1.15$ (SE). $36.6 \pm 0.0134$ (SE) and $36.3 \pm 0.0237 \%$ of the ICs were classified as noise by FIX for adults and children, respectively. These proportions of noise ICs were comparable to or smaller than in previous studies (Smith et al., 2009, 2013; Kelly et al., 2010; Rummel et al., 2013; Geranmayeh et al., 2014; Griffanti et al., 2014). There was no significant difference in a proportion of noise ICs between adults and children $\left(t_{(29.1)}=0.131, p=0.896, d=0.0424\right.$; Welch's $t$ test).

To obtain data free from nuisance signals such as artifact related to head motion and physiological and MR scanner noise, the time series of the noise ICs, the 12 motion parameters, the most dominant three principal components estimated from signals at lateral ventricles (Behzadi et al., 2007), a local time series of white matter estimated by ANATICOR (Jo et al., 2010), a trend of the preprocessed data, and a time series representing censored time points were regressed out from the preprocessed data. Adjacent TRs in which motion exceeded $0.35 \mathrm{~mm}$ were censored out. $6.29 \pm 3.08$ (SE) and $14.8 \pm 3.95 \%$ of volumes were censored out in adults and children, respectively. There was no significant difference in a proportion of volumes censored out between adults and children $\left(t_{(36.1)}=-1.700, p=0.0976, d=-0.530\right.$; Welch's $t$ test $)$ nor no significant relationship between proportions of volumes censored out and days of age within the child group $[r=-0.328, p=0.407$; the bias-corrected accelerated (BCa), bootstrap test on Pearson correlation].

Functional connectivity analysis. A subject-level GLM was applied to the preprocessed resting-state data to obtain individual measures of FC. For each ROI separately, the BOLD time series averaged within a ROI (see above, ROI identification) and time series representing censored time points were regressed against the whole-brain signal to quantify FC as the regression coefficient.

The group-level analysis was conducted individually for adults and children and also across the two groups. For the group-level adult and the group-level children analyses, the relationship between FC and the amount of adaptation (see above, Behavioral data analysis) was assessed using a mixed-effects model that included individual FC (regression coefficients and their variabilities, $t$ statistics) along with the amounts of adaptation as covariates. For the across-groups analysis, the relationship between development and FC related to speech learning was assessed in the mixed-effect model with covariate interaction. Specifically, we compared strengths of AAF-related FC, which is the slope of FC against the amount of adaptation, between adults and children by testing the difference in the effect of the covariate. To assess the statistical reliability of AAF-related FC measures, a non-Gaussian spatial ACF for each subject was averaged at the group level and then used in 3dClustSim to obtain nearest-neighbor, face-touching, two-sided cluster thresholds via a Monte Carlo simulation. The multiple comparisons were performed with two-tailed voxel-wise $p<0.002$ and cluster significance level of $\alpha<0.05 / 21$, which was adjusted in terms of the number of ROIs $(N=21)$ by Bonferroni correction.

Psychophysiological interaction analysis. To better distinguish auditory from somatosensory sources of AAF-related FC in our resting-state dataset, we conducted further tests that applied the generalized form of the context-dependent psychophysiological interaction (PPI) analysis (McLaren et al., 2012) to two task-based datasets. One dataset came from the speech production localizer task of the present study, and the other, which involved simple motor tasks, was taken from first 20 subjects of the preprocessed 1200 Subject Release of Human Connectome Project (HCP S1200; Van Essen et al., 2012; Barch et al., 2013). The speech task in our study required subjects to listen to words and repeat them aloud. The simple movements in the Connectome dataset involved repetitive hand, foot, and tongue movements. The speech task differs from the simple movements (apart from the specific body parts involved) in that the speech task recruits the auditory system as well as the basic somatomotor areas that are involved in simple motor task. By comparing the patterns of connectivity in the two tasks, we sought clues to whether connectivity in our own resting-state dataset was related to somatomotor or auditory function in speech. This analysis was only conducted for adults.

The 20 subjects of HCP S1200 data (11 males and 9 females) were ages $22-35$ years and had no prior neurologic disorders. The imaging data were acquired on a Siemens Skyra 3-tesla MRI scanner with gradients customized for the HCP and a 32-channel head coil (TR $=720 \mathrm{~ms}$; $\mathrm{TE}=33.1 \mathrm{~ms}$; slices $=72$; thickness $=2.0 \mathrm{~mm}$ isotropic with no gap; $\mathrm{mul}-$ tiband acceleration factor $=8$; see details in Ugurbil et al., 2013). The simple motor task consisted of four blocks of hand movements, four blocks of foot movements, and two blocks of tongue movements. In each block, subjects were presented with visual cues for $3 \mathrm{~s}$ and then tapped their left or right fingers, squeezed their left and right toes, or moved their tongue for $12 \mathrm{~s}$. Two 284-volume recordings were obtained. The data that had been registered in the Montreal Neurologic Institute space by HCP preprocessing were warped into Talairach space and spatial smoothed with FWHM $5 \mathrm{~mm}$ as we did for our own data (see above, Imaging data preprocessing).

PPI analysis was applied to the preprocessed data on a per-subject basis for each dataset. To obtain seed and PPI regressors that cannot be accounted for by task-evoked responses, we conducted a first of two GLM analyses that included regressors for experimental design convolved with basis functions (AFNI's SPMG2 for the speech task and AFNI's dmUBLOCK for the simple motor task), the 12 motion parameters, a polynomial function accounting for a trend signal, the first three principal components estimated from signals in the lateral ventricles, 


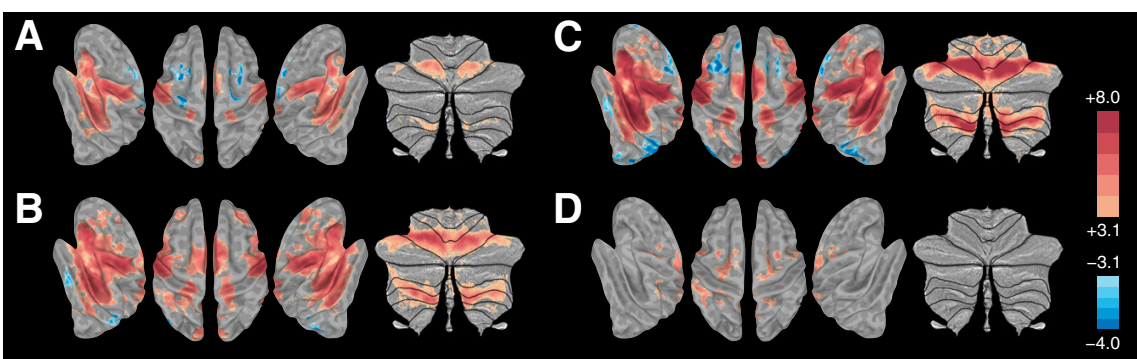

Figure 2. $\boldsymbol{A}-\boldsymbol{C}$, Brain activation in speech perception and production in children $(\boldsymbol{A})$, adults $(\boldsymbol{B})$, and data together $(\boldsymbol{C})$. D, Difference in activity between adults and children, adults-children. Each panel shows z-values resulting from volume-based analysis projected onto a cortical surface model (Saad et al., 2005) and cerebellum flat map (Diedrichsen and Zotow, 2015) for visualization purposes.

and the locally averaged time series of white matter estimated using ANATICOR. The seed regressor was the time course of the residual signal obtained from the first GLM averaged over the seed. To construct PPI regressors, the seed regressor was deconvolved with AFNI's SPMG1 basis function, multiplied with experimental design, and reconvolved with the basis function. We then conducted a second GLM analysis that included the seed and PPI regressors as well as all regressors of the first GLM. Resultant coefficients of PPI regressors represent changes in FC strength that were caused by task but cannot be accounted for by the response directly evoked by task. PPI effects of interest were assessed as the mean coefficients of PPI regressors over the region, which showed significant AAF-related FC with the seed (see highlighted area in Figs. 3, 4; see Results, Neural substrates of behavioral plasticity in speech audiomotor adaptation).

In the group-level analysis, the mean PPI effects were tested against zero using BCa bootstrap one-sample tests followed by the BonferroniHolm correction for the number of PPIs of interest $(N=16 ; 2$ tasks $\times 8$ FC). Among the samples included in 16 tests (24 samples of the speech task $\times 8$ tests +20 samples of the simple motor task $\times 8$ tests), three were beyond 2.8 SDs from the mean of each test and were excluded from the analysis.

\section{Results}

The present study examined the development of neural networks underlying the behavioral plasticity observed in speech sensorimotor learning. Intrinsic brain activity in two age groups, 5-12 and 18-30 years old, was measured before speech audio-motor adaptation (Fig. 1B). We assessed the relationships among brain networks, learning performance and development.

\section{Behavioral performance}

Subjects in both of the adult and child groups produced lower $\mathrm{F}_{1}$, more "bib"-like, sounds to compensate for an upward shift of $\mathrm{F}_{1}$ in auditory feedback, which makes "beb" more "bab"-like (trials $56-100$ in Fig. $1 B$ ). This adaptive response to feedback alteration was measured as changes in $\mathrm{F}_{1}$ relative to baseline. The amount of final adaptation achieved at trials 71-100 was on average 6.39 and $8.30 \%$ of baseline $F_{1}$ in adults and children $(\sim 27$ and $32 \%$ of the magnitude of the perturbations), respectively. $79.2 \%$ of adults and $73.7 \%$ of children were found to have adapted to altered feedback. When the feedback alteration was removed, adults gradually returned to baseline speech sounds, whereas children kept producing more "bib"-like sounds (trials 101-115 in Fig. $1 B$ ). Changes in $\mathrm{F}_{1}$ relative to the baseline observed in the last five washout trials were 3.59 and $7.74 \%$ in adults and children, respectively.

A series of $t$ tests revealed that the amount of adaptation in each of adults and children was significantly different from zero $t_{(23)}=5.83, p<0.0001, d=1.19$ for adults; $t_{(18)}=5.41$, $p=0.000192, d=1.24$ for children; $p$ values are corrected) and the amounts of adaptation did not significantly differ between the two groups $\left(t_{(34.1)}=1.02\right.$, uncorrected $p=0.316, d=0.320)$. The amount of washout was significantly different from zero in adults but not in children $\left(_{(23)}=2.84\right.$, $p=0.0375, d=0.579$ for adults; $t_{(17)}=$ 0.746, $p=0.866, d=0.176$ for children; $p$ values are corrected). The difference in the amount of washout between the two groups was not reliable $\left(t_{(28.3)}=0.795\right.$, uncorrected $p=0.433, d=0.260)$. There were no reliable differences between adults and children in a proportion of subjects who showed adaptive responses $\left(\chi_{1}^{2}=\right.$ $0.179, p=0.673, h=0.129)$. There was also no reliable relationship between the amount of final adaptation and days of age for children $(r=0.0794, p=0.699$; BCa bootstrap test on Pearson correlation).

A larger trial to trial fluctuation in $\mathrm{F}_{1}$ values was observed in children (Fig. 1B). We tested for a relationship between variability in $\mathrm{F}_{1}$ and adaptation performance. There was no reliable relationship between variability in $\mathrm{F}_{1}$ in the baseline and the amount of adaptation in either adults or children $(r=-0.134, p=0.636$ for adults; $r=0.000767, p=0.850$ for children; BCa bootstrap test on Pearson correlation). Moreover, there was no reliable difference in within-group variability in the amount of adaptation between the two groups $\left(F_{(18,23)}=1.56, p=0.315\right)$. This suggests that there is no relationship between variability of speech motor control and speech audio-motor adaptation.

Attention to the speech task as assessed using RT may account for between-subject differences in the amount of adaptation. To test for this possibility, a partial correlation analysis that controlled for subjects' age was conducted. A reliable relationship between the amount of final adaptation and mean RT was not found in either adults or children $\left(r=0.0749, t_{(21)}=0.344\right.$, $p=0.734$ for adults; $r=0.149, t_{(16)}=0.603, p=0.555$ for children). This suggests that differences in adaptation behavior that were observed in this study are unlikely to be accounted for by differences in concentration on the task, as assessed using RT.

Overall, both adults and children successfully adapted to the auditory feedback alteration, and their adaptive responses were similar. This indicates that any differences in brain activity measures between adults and children cannot be accounted for by differences in adaptation performance.

\section{Brain activity differences in speech perception and production}

We measured brain activity in speech perception and production to identify regions of interest for the resting-state analyses. Figure 2, $A$ and $B$, show brain activity during the localizer session in which adults and children were listening to the sounds of words and repeating these once each inside the scanner. Positive BOLD responses were observed in diverse regions including inferior prefrontal gyrus, insula, sensorimotor cortex related to speech articulation and vocalization, auditory cortex, occipital cortex, thalamus, putamen, and cerebellum. Negative BOLD responses relative to baseline activity were observed in the inferior parietal lobe of adults and in the superior frontal sulcus of children. Based on these responses and the findings of previous speech production and perception studies (Tourville et al., 2008; Rauschecker and Scott, 2009; Hickok, 2012; Niziolek and 


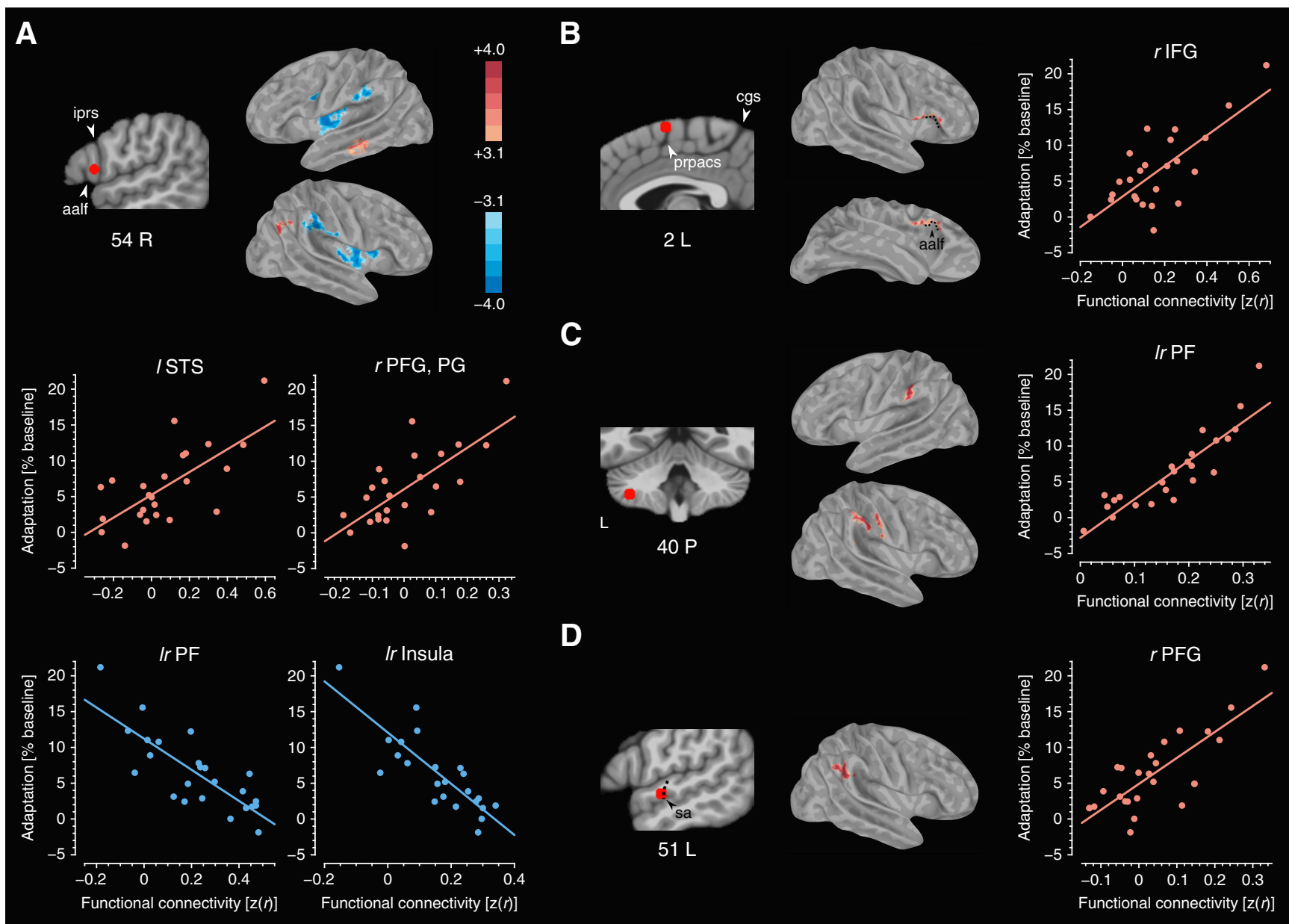

Figure 3. FC patterns in which the strength of the connectivity was significantly correlated with the amount of adaptation in adults. $\boldsymbol{A}-\boldsymbol{D}$, Red circles superimposed onto volume images indicate locations of right IFG $(\boldsymbol{A})$, pre-SMA $(\boldsymbol{B})$, left CbVIII $(\boldsymbol{C})$, and left aSTS ROIs (D). Highlighted color on cortical surface maps represent $z$-values within clusters detected by the analysis. Scatter plots show linear relationships between the amount of adaptation and mean FC over the detected cluster. Image labels such as $54 R$ indicate the right side of the brain $54 \mathrm{~mm}$ from the midline. aalf, Anterior ascending ramus of the lateral fissure; iprs, inferior precentral sulcus; prpacs, preparacentral sulcus; cgs, cingulate sulcus; sa, sulcus acousticus.

Guenther, 2013; Zheng et al., 2013; Johnson et al., 2019; Floegel et al., 2020), we selected ROIs for subsequent analysis from the activity observed in the across-group analysis, as shown in Table 1.

The overall pattern of brain activity in the listen and repeat localizer task was basically similar for the two groups, but the taskrelated response of children was weaker, and its spatial extent was more restricted than that of adults. Figure $2 D$ shows a comparison of speech-related responses between adults and children. Differences in BOLD responses between the groups were observed in dorsal premotor and superior parietal areas. No significant differences were observed in the other regions, in particular speech sensorimotor circuits, which suggest that activity related to speech production was comparable in children and adults. There was no region in which the magnitude of the taskrelated response was correlated with the amount of adaptation neither for adults nor children (voxel-wise $p<0.002$, nonGaussian ACF corrected cluster significant level $\alpha>0.05$ ), implying that there is no relationship between feedback-based adaptation performance and the brain activity observed in speech production under conditions of veridical feedback.

To carry out statistical comparisons of the imaging data in a common space, the images of children as well as those of adults were aligned to an adult brain template. To assess a potential bias caused by this mismatch, we compared the accuracy of the registration from individual brains to the template brain for adults and children separately. The similarity in the location of the sulci of the template brain and those of the individual brains warped onto the template brain was quantified with the Dice coefficient. The Dice coefficient was on average $0.581 \pm 0.00667$ (SE) and $0.581 \pm 0.00791$ for adults and children, respectively. There was no reliable difference between adults and children $\left(t_{(40.5)}=0.0398, p=0.968, d=0.0120\right)$, indicating that the nonlinear registration worked as well for children as for adults.

\section{Neural substrates of behavioral plasticity in speech audio- motor adaptation}

To identify the neural substrates of the behavioral plasticity observed in each of the adults and children, we detected brain areas in which resting-state FC measures were correlated with the amount of adaptation. ROIs were derived from task-related responses in an across-group analysis (Table 1). ROIs were tested separately for each hemisphere.

Figure 3 focuses on adults. It shows for each seed region those clusters of voxels whose FC values were significantly correlated with the amount of adaptation. The main finding was the presence of significant relationships linking right IFG and associative sensory regions of the brain. Specifically, it was observed that FC between right IFG (area 44) and both left aSTS and right posterior supramarginal gyrus and anterior angular gyrus (PFG/PG; 


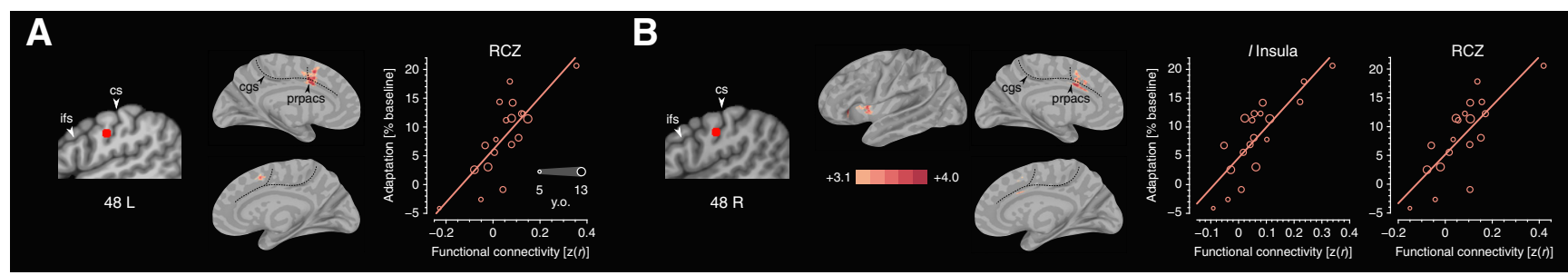

Figure 4. FC patterns in which the strength of connectivity is significantly correlated with the amount of adaptation in children. $\boldsymbol{A}, \boldsymbol{B}$, Red circles superimposed onto volume images indicate locations of left S1/M1 (A), and right S1/M1 R0ls $(\boldsymbol{B})$. Highlighted color on cortical surface maps represents z-values within clusters detected by the analysis. Scatter plots show linear relationships between the amount of adaptation and mean FC over the cluster, with individual values given as circles whose size corresponds to the age of the children. ifs, Inferior frontal sulcus; cs, central sulcus.

von Economo and Koskinas, 1925; Margulies and Petrides, 2013; Petrides, 2014) were positively correlated with the amount of adaptation (Fig. $3 A$; cluster size $=9.28 \mathrm{~mm}^{3}, r=0.674, p<0.0001$ for left aSTS; cluster size $=9.67 \mathrm{~mm}^{3}, r=0.692, p<0.0001$ for right PFG/PG). In addition, FC between right IFG and each of bilateral $\mathrm{PF}$ and anterior insula negatively predicted individual differences in the amount of adaptation (Fig. $3 A$; cluster size $=11.7$ and $12.9 \mathrm{~mm}^{3}$ for left and right hemispheres, $r=-0.791$, $p<0.0001$ for PF; cluster size $=14.4$ and $15.0 \mathrm{~mm}^{3}$ for left and right hemispheres, $r=-0.815, p<0.0001$ for insula). Connectivity values, which positively predicted learning, were also observed in FC between pre-SMA and right IFG around the anterior ascending ramus of the lateral fissure (areas 44 and 45), between left CbVIII and bilateral PF, and between left aSTS and right PFG (Fig. 3B-D; cluster size $=9.73 \mathrm{~mm}^{3}, r=0.705, p<0.0001$ for pre-SMA; cluster size $=10.3$ and $11.8 \mathrm{~mm}^{3}$ for left and right hemispheres, $r=0.898$, $p<0.0001$ for left CbVIII; cluster size $=10.9 \mathrm{~mm}^{3}, r=0.772$, $p<0.0001$ for left aSTS). These patterns of AAF-related FC suggest that right area 44 works together with associative sensory regions distributed over the brain to calibrate the mapping between speech sounds and articulations.

A separate analysis for children detected patterns of AAFrelated FC that were different from those in adults. In children, FC between bilateral S1/M1 and each of posterior rostral cingulate zone (RCZ) and left anterior insular cortex were positively correlated with the amount of adaptation (Fig. 4; cluster size $=10.3 \mathrm{~mm}^{3}, r=0.780, p<0.0001$ for left S1/M1-RCZ; cluster size $=10.2 \mathrm{~mm}^{3}, r=0.764, p=0.00115$ for right S1/M1-RCZ; cluster size $=10.2 \mathrm{~mm}^{3}, r=0.824, p<0.0001$ for right S1/M1insula). There were no other significant relationships between connectivity and learning in either children or adults.

We conducted additional analyses to rule out the possibility that the different patterns observed in children and adults might be related to factors such as movement in the scanner. This possibility was assessed by testing the relationship between the average motion as measured in AFNI and amount of the adaptation. There was no significant correlation between these two parameters $(r=0.181$, $p=0.385$; BCa bootstrap test on Pearson correlation). We also tested the relationship between the amount of the adaptation and the average FC over the detected clusters including each subject's average motion as a confound. Even after accounting for differences between subjects in movement, all of the relationships shown in Figure 4 were still reliable $\left(r=0.757, t_{(16)}=4.64, p=0.000275\right.$ for left S1/M1-RCZ; $r=0.778, t_{(16)}=4.96, p=0.000142$ for right S1/M1-RCZ; $r=0.836$, $t_{(16)}=6.10, p<0.0001$ for right S1/M1-insula; Spearman partial correlation). These two analyses argue against the possibility that AAFrelated FC patterns in children were because of cortical activity associated with body movements during the MR scans.

The separate analyses reported above for adults and children raise the possibility that the mature capacity for speech adaptation involves interactions between right area 44 and associative sensory areas, whereas the immature architecture of the child's brain relies on more basic somatic and motor regions to produce the recalibration needed to acquire a novel audio-motor association. This idea was tested in an analysis that involved a direct comparison of differences in the strength of AAF-related FC between adults and children. Significant differences between adults and children were observed in AAF-related FC between right IFG and each of right $\mathrm{PFG} / \mathrm{PG}$ and right $\mathrm{PF}$, between preSMA and right IFG, and between S1/M1 and each of posterior RCZ and left insula (Fig. 5). Subsequent correlation analyses between the amount of adaptation and mean FC within the detected cluster revealed distinct patterns for children and adults in the relationship between adaptation and FC. Specifically, except for connectivity related to right IFG, the sign of the associations between connectivity and adaptation was opposite for children and adults, and these correlations were significant within each group (Table 2).

We reasoned that if the oppositely signed correlation between the amount of adaptation and the strength of connectivity is a matter of development, then the AAF-FC correlations that are observed in children should be closer to those of adults in older children. As a preliminary test of this idea, we split the children into two groups, with subjects being either younger or older than 9 years old ( $N=8$ and 11 for younger and older). We then computed correlations between AAF and FC with each of pre-SMA and bilateral S1/M1 as seed regions because these areas showed a significant change in sign in connectivity patterns between children and adults.

As expected, younger children showed steeper AAF-FC relationships in which the signs were opposite to those of adults (Fig. $6)$. Steeper negative relationships were observed in younger children in FC of pre-SMA to IFG $(r=-0.834$ and -0.262 , $p=0.0192$ and 0.399 for younger and older children; BCa bootstrap test on the Pearson correlation). Steeper positive relationships were also obtained in younger children in FC of S1/M1RCZ and S1/M1-insula $(r=0.848$ and 0.566, $p=0.00903$ and 0.0520 for left S1/M1-RCZ of younger and older children; $r=0.826$ and $0.482, p=0.0328$ and 0.111 for right S1/M1-RCZ; $r=0.934$ and $0.260, p=0.00311$ and 0.329 for right S1/M1-insula, again for younger and older children; BCa bootstrap test on Pearson correlation). The fact that increasingly adult-like patterns of connectivity are observed in the oldest children suggests that associations between behavioral plasticity in speech learning and brain networks evolve in late childhood.

\section{Somatosensory versus auditory contributions to AAF-related} FC

We found that in adults, resting-state FC between area 44 in the right IFG and diverse associative regions of the brain is related to 


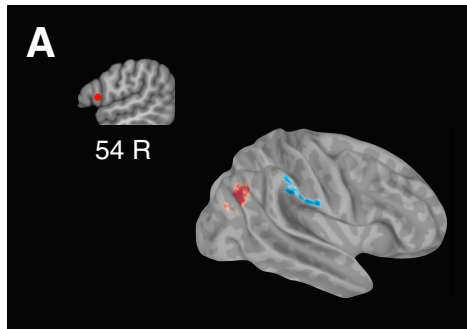

Ad. $>$ Ch. $3.1 \square 4.0$

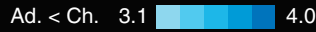

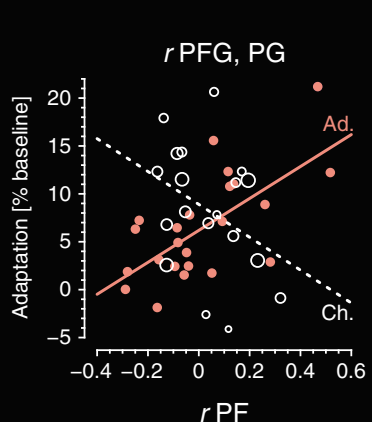

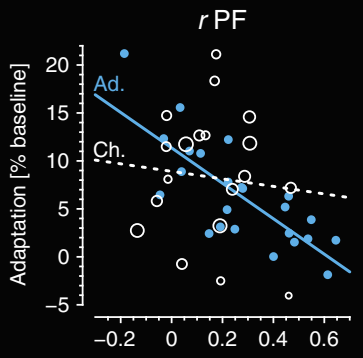

Functional connectivity $[\mathrm{z}(r)]$ c
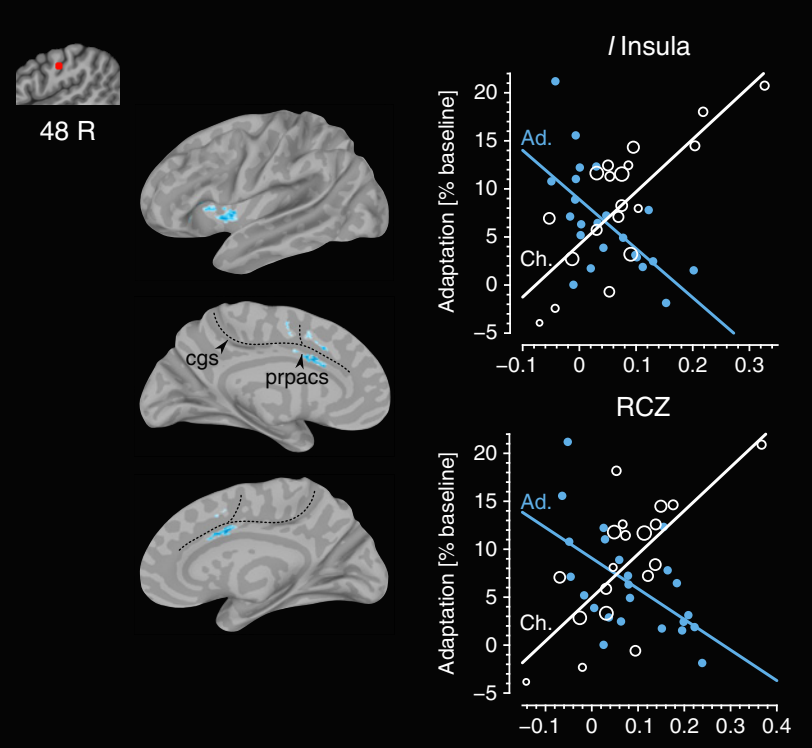

Functional connectivity $[z(r)]$

\section{B}

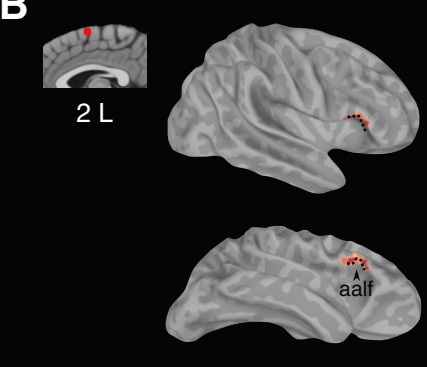

$r \mathrm{IFG}$

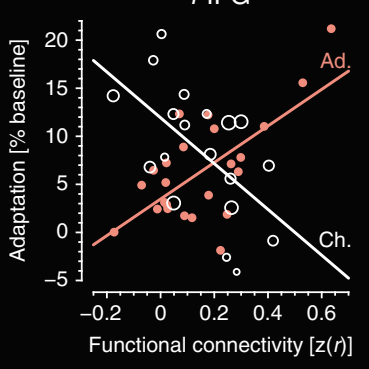

D

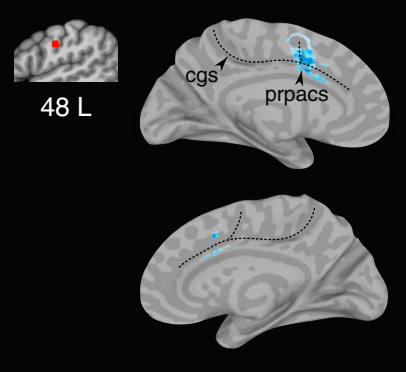

$\mathrm{RCZ}$

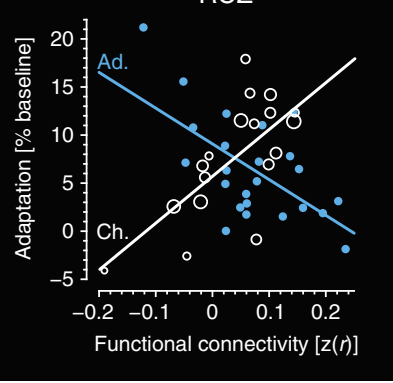

Figure 5. Regions in which the strength of the relationship between $\mathrm{FC}$ and adaptation was significantly different between adults (Ad.) and children (Ch.). $\boldsymbol{A}-\boldsymbol{D}$, Red circles superimposed onto volume images indicate locations of right IFG (A), pre-SMA (B), left S1/M1 (C), and right S1/M1 ROIs (D; Figs. 3 and 4 show larger pictures). Highlighted color on cortical surface maps represents $z$-values within clusters detected by the analysis. Scatter plots show linear relationships between the amount of adaptation and mean $\mathrm{FC}$ over the cluster, with individual values given as circles whose size corresponds to the ages of the children. Significant and nonsignificant relationships are denoted with solid and dashed lines, respectively $(p<0.01 ;$ Table 2).

speech audio-motor adaptation. To interpret the functional roles of these FC patterns, we examined in a PPI analysis how FC is modulated in our speech task and in a simple motor task. We reasoned that the resting-state connectivity patterns in our data, which are also seen in the PPI analysis of simple motor tasks, might reflect effects related to motor efferent and somatosensory afferent activity. In contrast, connectivity patterns seen in the PPI analysis of the speech motor task may reflect audio-motor effects.

The PPI connectivity patterns of right IFG differed for the two tasks. Connectivity with each of bilateral PF and insula were observed in the simple motor task, whereas PPI connectivity with right PFG/PG and left aSTS were seen in the speech motor task (Fig. $7 A ; p=0.0211$ for PF in motor; $p=0.0211$ for insula in motor; $p<0.0001$ for PFG/PG in speech; $p=0.0113$ for aSTS in speech; $p>0.05$ for the others; $p$ values are corrected). This suggests that the former connectivity patterns are primarily somatic, whereas the latter reflect auditory-related connectivity. In the resting-state data presented above, individuals with weaker functional connectivity between area 44 and each of PF and insula (which are both somatic) show better adaptation, whereas those with greater connectivity between area 44 and each of PFG/PG and aSTS (both auditory) showed better adaptation (Fig. $3 \mathrm{~A}$ ). The differing patterns of PPI connectivity of IFG in the two tasks suggests that associative somatosensory areas ( $\mathrm{PF}$ and insula)
Table 2. Resting state FC of which strength of coupling with the amount of adaptation was significantly different between adults and children

\begin{tabular}{|c|c|c|c|c|c|c|c|c|c|c|}
\hline \multirow[b]{2}{*}{ ROI } & \multicolumn{6}{|l|}{ Cluster } & \multicolumn{2}{|l|}{ Adults } & \multicolumn{2}{|l|}{ Children } \\
\hline & Region & $\mathrm{RL}$ & AP & IS & $z$ & $\mathrm{~mm}^{3}$ & $r$ & $p$ & $r$ & $p$ \\
\hline \multirow[t]{2}{*}{$r$ IFG } & $\mathrm{rPFG} / \mathrm{PG}$ & -53 & 61 & 26 & 4.41 & 9.78 & 0.655 & $<0.0001$ & -0.352 & 0.077 \\
\hline & rPF & -61 & 19 & 24 & -3.95 & 12.2 & -0.769 & $<0.0001$ & -0.0816 & 0.664 \\
\hline Pre-SMA & $r I F G$ & -47 & -19 & 8 & 4.74 & 9.81 & 0.633 & 0.00135 & -0.567 & 0.00336 \\
\hline I S1/M1 & RCZ & 1 & -5 & 40 & -4.36 & 12.0 & -0.588 & 0.00296 & 0.756 & $<0.0001$ \\
\hline \multirow[t]{2}{*}{ r S1/M1 } & I Insula & 39 & -9 & 4 & -4.66 & 12.1 & -0.639 & 0.00104 & 0.782 & $<0.0001$ \\
\hline & $\mathrm{RCZ}$ & -1 & -13 & 34 & -4.81 & 10.6 & -0.569 & $<0.0001$ & 0.715 & $<0.0001$ \\
\hline
\end{tabular}

Coordinates are given in Talairach space in RAI order. $z$, Maximum $z$-value testing adults $>$ children within each cluster; $r$, correlation coefficient resulting from correlation analyses of the relationship between the amount of AAF and the average strength of $\mathrm{FC}$ within a corresponding cluster; $p, p$ value resulting from correlation analyses of the relationship between the amount of AAF and the average strength of $F C$ within a corresponding cluster; RL, right-left; AP, anterior-posterior; IS, inferior-superior; r, right; I, left.

interact with right area 44 in a manner that is opposite to that of associative auditory areas (PFG/PG and aSTS) possibly to tolerate somatic error for successful adaptation to altered auditory feedback.

Further PPI related measures are as follows: Connectivity between pre-SMA and right IFG and between left CbVIII and bilateral PF was observed in both of the tasks (Fig. $7 B, C$; $p<0.0001$ for pre-SMA-IFG in motor; $p=0.00965$ for preSMA-IFG in speech; $p=0.00123$ for CbVIII-PF in motor; 

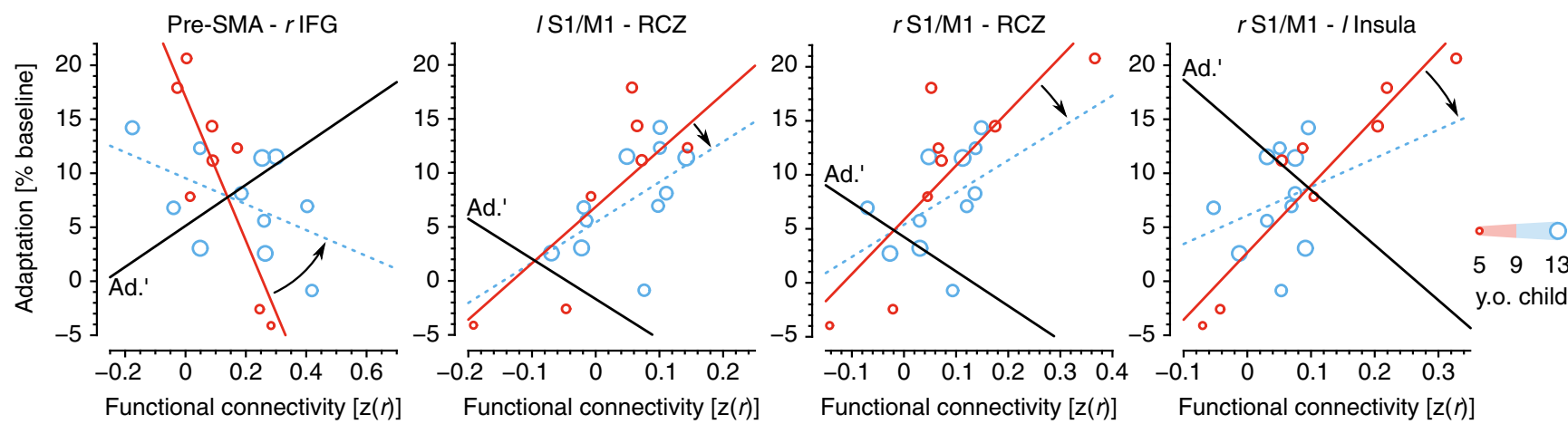

Figure 6. Relationship between the strength of functional connectivity and the amount of adaptation in children ages 5-9 years (red) and ages 9-12 years (cyan). Individuals are shown as circles whose sizes correspond to the age of the children. Significant and nonsignificant relationships are denoted with solid and dashed lines, respectively. Black lines (Ad.') show the relationship that was observed in adults. To visualize changes in the slopes of the lines, the black line in each panel is aligned to cross at the intersection between red and cyan lines.

A

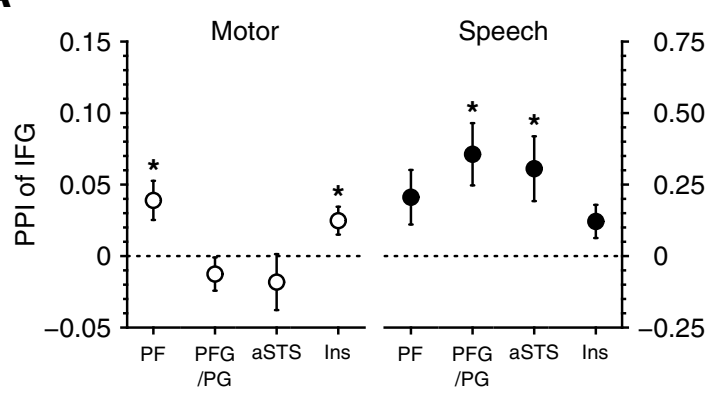

C

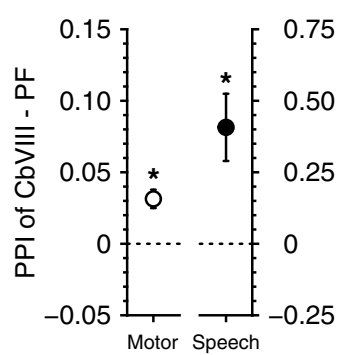

B

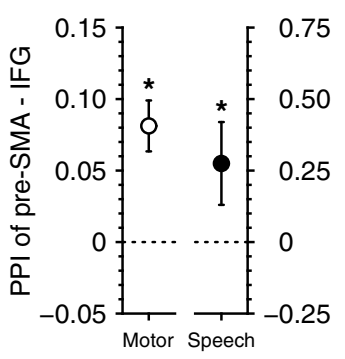

E

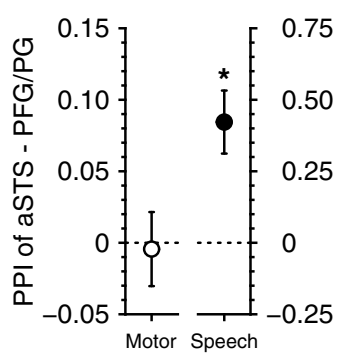

which involve long-range intercortical connections, undergo remodeling through to early adulthood (Fair et al., 2007; Baum et al., 2020; Váša et al., 2020). The question asked in the present study is whether these later occurring changes that include those in associative cortices have implications for the capacity for human speech learning and adaptation. Our functional connectivity analysis of young adults indicated that the brain networks comprising transmodal associative regions, IFG, pre-SMA, PF, PFG/PG, and aSTS, were related to speech adaptation performance. The analysis of children indicated that the connectivity between S1/M1 and posterior RCZ, a motor area on the preparacentral sulcus, was also adaptation related. These different relationships observed in adults and children are anchor points (sensorimotor versus transmodal) in the brain's macroscale structure and its remodeling during development.

Our functional connectivity analysis showed that children and adults had opposite patterns of correlation between learning and functional connectivity. This flip may be associated with a previous finding that there are two distinct modes in the de-

$p=0.0112$ for CbVIII-PF in speech; $p$ values are corrected). Connectivity between left aSTS and right PFG and between bilateral S1/M1 and posterior RCZ was found in the speech task but not in the simple motor task (Fig. $7 D, E ; p<0.0001$ for aSTSPFG in speech; $p=0.00236$ for bilateral S1/M1-RCZ in speech; $p>0.05$ for the others; $p$ values are corrected).

\section{Discussion}

Primate cerebral cortex is organized in terms of a functional gradient that spans primary sensory and motor cortices and transmodal associative regions (Margulies et al., 2016) and extends to anatomic properties such as white-matter architecture (VázquezRodríguez et al., 2019; Baum et al., 2020), intercortical myelination (Huntenburg et al., 2017; Burt et al., 2018), and laminar differentiation (Paquola et al., 2019). Within this macroscale organization, basic sensory and motor regions mature in early childhood, whereas frontoparietal and default mode networks, velopment of functional connectivity-conservative regions, corresponding to basic sensory and motor cortices, are the regions in which connections are already strong by early adolescence and strengthen further in young adults, whereas mutable regions, corresponding to associative cortices, are the regions in which connections are initially strong and weaken in adulthood (Váša et al., 2020). We found the neurobehavioral patterns of children in conservative regions and the patterns of adults in mutable regions, suggesting that reconfigurations of cortex may lead the distinct encoding patterns of speech learning in the developing brain. Together, the distinct patterns observed across the two age groups suggest that human abilities to learn speech are built on cortical remodeling that is observable in late childhood and is stabilized in adults.

Reciprocal connections between ventrolateral prefrontal/premotor cortex and sensory systems are central to speech sensorimotor learning (Rauschecker and Scott, 2009; Houde and 
Nagarajan, 2011; Tourville and Guenther, 2011; Hickok, 2012). In the present study, we observed that connectivity between area 44 and each of PFG/PG and aSTS was positively related to the amount of subsequent adaptation and connectivity between area 44 and PF was negatively related. These neural circuits are thought to be associated with the establishment of sensory targets and motor commands over the course of learning (Houde and Nagarajan, 2011; Tourville and Guenther, 2011). In particular, it has been proposed that right IFG serves to translate errors in auditory feedback detected in associative auditory regions into corrective motor commands (Tourville and Guenther, 2011; Floegel et al., 2020). Imaging studies have found that right IFG activity is suppressed during normal speech production, which presumably relies less on error-based processes (Blank et al., 2002), whereas the activity increases during speech with AAF (Tourville and Guenther, 2011; Johnson et al., 2019; Floegel et al., 2020). Inferior frontal gyrus responds to speech feedback perturbations concurrently with superior temporal regions distributed from anterior to posterior and parietal regions including PF, PFG or PG (Tourville et al., 2008; Niziolek and Guenther, 2013; Zheng et al., 2013; Johnson et al., 2019; Floegel et al., 2020), and functional connectivity among these regions changes in association with adaptation (Floegel et al., 2020). Transcranial magnetic stimulation and aphasia studies showed disruption of PF, and PFG degrades speech audio-motor control (Shum et al., 2011; Rogalsky et al., 2015; Behroozmand et al., 2018).

Area 44 was found to be functionally connected with the insula. Adaptation varied inversely with connectivity strength. Imaging and aphasia studies have observed insular involvement in aspects of speech production such as speech planning (Dronkers, 1996), motor coordination (Ackermann and Riecker, 2004), and feedback processing (Kleber et al., 2013, 2017; Woolnough et al., 2019). In particular, an interaction of auditory and somatosensory feedback was observed in the insula-its activity during singing was downregulated by anesthesia of the vocal folds and upregulated by masking sung sounds (Kleber et al., 2013, 2017). The result of our PPI analysis, which indicated that connectivity between area 44 and insula is somatic, suggests that somatosensory aspects of insular activity may tap into the cortical speech circuit via this connection.

It was observed that functional connectivity between a preSMA seed and IFG, areas 44 and 45, was related to speech audiomotor adaptation. Presupplementary motor area is known to contribute to movement sequencing (Kennerley et al., 2004), learning of sensorimotor associations (Loh et al., 2020), sensorimotor imagery (Lima et al., 2016), and encoding auditory and somatosensory information in working memory (Vergara et al., 2016). Given the potential role of the IFG in updating motor commands, pre-SMA in combination with IFG may contribute to learning-related reorganization of speech movement sequences. Alternatively, IFG may need auditory and somatosensory information encoded by pre-SMA in working memory to establish sensory targets for speech movements. This idea comes from previous findings on the relationship between working memory capacity and sensorimotor control in speech and limb movements (Bo and Seidler, 2009; Guo et al., 2017; Sidarta et al., 2018; Ito et al., 2020). Indeed, the IFG region that we found to work with the pre-SMA seed is in the area 45 region, where previous studies found activity associated with visual, somatosensory, and auditory memory retrieval in delayed match-to-sample tasks (Kostopoulos and Petrides, 2003, 2016; Kostopoulos et al., 2007).

In domains others than speech, the cerebellum is thought to take part in sensory-motor mapping by predicting sensory consequences of motor commands (Bodranghien et al., 2016). A previous speech study showed that cerebellar degeneration selectively impairs adaptation to AAF that is introduced predictably, suggesting that cerebellum may also be related to predictive aspects of sensorimotor control in speech (Parrell et al., 2017). In the present study, we found that connectivity between PF and the CbVIII seed was related to adaptation to predictable AAF. There are a number of studies that support the contribution of this connection to sensorimotor adaptation. Cerebellar lobule VIII activity has been observed in altered auditory and somatosensory feedback in speech (Tourville et al., 2008; Golfinopoulos et al., 2011). The speech somatosensory feedback study further observed concurrent PF and CbVIII activity (Golfinopoulos et al., 2011). Rostral inferior parietal lobule, PF and PFG, are the targets of output from cerebellum (Bostan et al., 2013). This may imply that the connection between PF and CbVIII that was observed here is involved in the mapping between motor and somatosensory information in speech production.

In children, we observed that functional connectivity between posterior RCZ, which is rostral to the preparacentral sulcus, and the S1/M1 seeds was associated with differences in speech audiomotor adaptation. Human cingulate cortex is subdivided into three regions, caudal cingulate zone, posterior RCZ, and anterior RCZ (Picard and Strick, 1996). Although these three regions have anatomic connections with the spinal cord, premotor, M1, and prefrontal cortex, the more caudal part has denser connections with the spinal cord and motor cortex. Of these three regions, only posterior and anterior RCZ have face motor representations (Amiez and Petrides, 2014). Posterior and anterior RCZs are associated with control motor behaviors and feedback monitoring, respectively (Picard and Strick, 2001; Morecraft and Tanji, 2009). In speech, the RCZ region is involved in coding errors in auditory feedback specifically during speech production (Zheng et al., 2013). Prior experience in audio-motor control increases RCZ activity during compensation for pitch perturbations (Zarate and Zatorre, 2008). These findings indicate that RCZ is related to speech sensorimotor control at the level of function and individual traits. Together, RCZ-S1/M1 connectivity may directly influence articulatory movements based on auditory feedback at a fine control level.

In contrast to differences in learning-related brain connectivity between adults and children, there was little or no difference in audio-motor adaptation between these two groups, which is consistent with previous work (Shiller et al., 2010; MacDonald et al., 2012; Daliri et al., 2018; Caudrelier et al., 2019; Kim et al., 2020). However, unlike adults, children showed persistent adaptation during washout trials. In work on human limb movement, sensorimotor adaptation is thought to be a mixture of implicit processes that bring slow and persistent behavioral change and explicit processes that result in fast but transient changes (Smith et al., 2006). There is evidence that implicit processes in motor learning may mature earlier in human development than explicit processes (Vasudevan et al., 2011; Rossi et al., 2019). It is thought that speech motor learning predominantly relies on implicit processes (Munhall et al., 2009). Nevertheless, given previous work on limb movement, the absence of washout in children may result from the later development of explicit processes that could change behaviors quickly.

The present work advances our understanding of the neural mechanisms of multisensory integration in motor learning. Multisensory integration in speech learning was hypothesized to be a factor contributing to individual differences in susceptibility to delayed auditory feedback (Yates, 1965). In a case of AAF, 
auditory feedback is shifted away from auditory target by the perturbation, whereas somatosensory feedback initially remains within the somatosensory target zone. However, over the course of adaptation to AAF, somatosensory feedback in turn deviates from its prelearning target. This trade-off between auditory and somatosensory feedback was observed behaviorally where individuals who adapted to AAF during speech production failed to adapt to altered somatosensory feedback (Lametti et al., 2012). The neural mechanisms that underlie the variability in sensory preference in motor learning remain uncertain.

The combination of functional connectivity and PPI analyses on separate datasets here demonstrated that right area 44 has two sets of AAF-related connections. The connections of area 44 with PF and insula are predominantly somatic, and a weaker connection predicted a larger amount of adaptation. The connections of area 44 with PFG/PG and aSTS are predominantly auditory, and, in this case, a stronger connection predicted a larger amount of adaptation. These two sets of the connections of area 44 may be the neuronal homolog of the behavioral finding of sensory preference mentioned above. These connections may also contribute to typical speech production, even without external perturbations, as two of the regions, PF and STS, are known to have inversely correlated levels of activation across individuals during overt picture naming (Seghier et al., 2015).

In the context of development, it is noteworthy that sensory preference depends on sensory experience as a previous study showed that although all postlingually deaf subjects reliably adapted to altered somatosensory feedback during speech production, one third of normally hearing subjects do not (Nasir and Ostry, 2008). The cortical speech circuit may be reorganized over the course of human development depending on one's activities and the surrounding environment. This biological adaptation may account for sensory preference and enable flexible but stable human speech abilities.

\section{References}

Ackermann H, Riecker A (2004) The contribution of the insula to motor aspects of speech production: a review and a hypothesis. Brain Lang 89:320-328.

Amiez C, Petrides M (2014) Neuroimaging evidence of the anatomo-functional organization of the human cingulate motor areas. Cereb Cortex 24:563-578.

Anderson N (1978) On the calculation of filter coefficients for maximum entropy spectral analysis. In: Modern spectrum analysis (Childers DG, ed), pp 252-255. Washington, DC: IEEE Computer Society.

Barch DM, Burgess GC, Harms MP, Petersen SE, Schlaggar BL, Corbetta M, Glasser MF, Curtiss S, Dixit S, Feldt C, Nolan D, Bryant E, Hartley T, Footer O, Bjork JM, Poldrack R, Smith S, Johansen-Berg H, Snyder AZ, Van Essen DC, et al. (2013) Function in the human connectome: taskfMRI and individual differences in behavior. Neuroimage 80:169-189.

Baum GL, Cui Z, Roalf DR, Ciric R, Betzel RF, Larsen B, Cieslak M, Cook PA, Xia CH, Moore TM, Ruparel K, Oathes DJ, Alexander-Bloch AF, Shinohara RT, Raznahan A, Gur RE, Gur RC, Bassett DS, Satterthwaite TD (2020) Development of structure-function coupling in human brain networks during youth. Proc Natl Acad Sci U S A 117:771-778.

Beckmann CF, Smith SM (2004) Probabilistic independent component analysis for functional magnetic resonance imaging. IEEE Trans Med Imaging 23:137-152.

Behroozmand R, Phillip L, Johari K, Bonilha L, Rorden C, Hickok G, Fridriksson J (2018) Sensorimotor impairment of speech auditory feedback processing in aphasia. Neuroimage 165:102-111.

Behzadi Y, Restom K, Liau J, Liu TT (2007) A component based noise correction method (CompCor) for BOLD and perfusion based fMRI. Neuroimage 37:90-101.

Blank SC, Scott SK, Murphy K, Warburton E, Wise RJS (2002) Speech production: Wernicke, Broca and beyond. Brain 125:1829-1838.
Bo J, Seidler RD (2009) Visuospatial working memory capacity predicts the organization of acquired explicit motor sequences. J Neurophysiol 101:3116-3125.

Bodranghien F, Bastian A, Casali C, Hallett M, Louis ED, Manto M, Mariën P, Nowak DA, Schmahmann JD, Serrao M, Steiner KM, Strupp M, Tilikete C, Timmann D, van Dun K (2016) Consensus Paper: revisiting the symptoms and signs of cerebellar syndrome. Cerebellum 15:369-391.

Boersma P, Weenink D (2018) Praat: doing phonetics by computer. Amsterdam: Phonetic Sciences, University of Amsterdam.

Bostan AC, Dum RP, Strick PL (2013) Cerebellar networks with the cerebral cortex and basal ganglia. Trends Cogn Sci 17:241-254.

Burt JB, Demirtaş M, Eckner WJ, Navejar NM, Ji JL, Martin WJ, Bernacchia A, Anticevic A, Murray JD (2018) Hierarchy of transcriptomic specialization across human cortex captured by structural neuroimaging topography. Nat Neurosci 21:1251-1259.

Caudrelier T, Ménard L, Perrier P, Schwartz J-L, Gerber S, Vidou C, RochetCapellan A (2019) Transfer of sensorimotor learning reveals phoneme representations in preliterate children. Cognition 192:103973.

Cox RW (1996) AFNI: software for analysis and visualization of functional magnetic resonance neuroimages. Comput Biomed Res 29:162-173.

Cox RW, Chen G, Glen DR, Reynolds RC, Taylor PA (2017) FMRI clustering in AFNI: false-positive rates redux. Brain Connect 7:152-171.

Daliri A, Wieland EA, Cai S, Guenther FH, Chang S-E (2018) Auditorymotor adaptation is reduced in adults who stutter but not in children who stutter. Dev Sci 21: e12521.

Darainy M, Vahdat S, Ostry DJ (2019) Neural basis of sensorimotor plasticity in speech motor adaptation. Cereb Cortex 29:2876-2889.

Diedrichsen J, Zotow E (2015) Surface-based display of volume-averaged cerebellar imaging data. PLoS One 10:e0133402.

Dronkers NF (1996) A new brain region for coordinating speech articulation. Nature 384:159-161.

Fair DA, Dosenbach NUF, Church JA, Cohen AL, Brahmbhatt S, Miezin FM, Barch DM, Raichle ME, Petersen SE, Schlaggar BL (2007) Development of distinct control networks through segregation and integration. Proc Natl Acad Sci U S A 104:13507-13512.

Fischl B (2012) FreeSurfer. Neuroimage 62:774-781.

Fitch WT, de Boer B, Mathur N, Ghazanfar AA (2016) Monkey vocal tracts are speech-ready. Sci Adv 2:e1600723.

Floegel M, Fuchs S, Kell CA (2020) Differential contributions of the two cerebral hemispheres to temporal and spectral speech feedback control. Nat Commun 11:2839.

Geranmayeh F, Wise RJS, Mehta A, Leech R (2014) Overlapping networks engaged during spoken language production and its cognitive control. J Neurosci 34:8728-8740.

Golfinopoulos E, Tourville JA, Bohland JW, Ghosh SS, Nieto-Castanon A, Guenther FH (2011) fMRI investigation of unexpected somatosensory feedback perturbation during speech. Neuroimage 55:1324-1338.

Griffanti L, Salimi-Khorshidi G, Beckmann CF, Auerbach EJ, Douaud G, Sexton CE, Zsoldos E, Ebmeier KP, Filippini N, Mackay CE, Moeller S, Xu J, Yacoub E, Baselli G, Ugurbil K, Miller KL, Smith SM (2014) ICAbased artefact removal and accelerated fMRI acquisition for improved resting state network imaging. Neuroimage 95:232-247.

Griffanti L, Douaud G, Bijsterbosch J, Evangelisti S, Alfaro-Almagro F, Glasser MF, Duff EP, Fitzgibbon S, Westphal R, Carone D, Beckmann CF, Smith SM (2017) Hand classification of fMRI ICA noise components. Neuroimage 154:188-205.

Guo Z, Wu X, Li W, Jones JA, Yan N, Sheft S, Liu P, Liu H (2017) Top-down modulation of auditory-motor integration during speech production: the role of working memory. J Neurosci 37:10323-10333.

Hickok G (2012) Computational neuroanatomy of speech production. Nat Rev Neurosci 13:135-145.

Houde JF, Jordan MI (1998) Sensorimotor adaptation in speech production. Science 279:1213-1216

Houde JF, Nagarajan SS (2011) Speech production as state feedback control. Front Hum Neurosci 5:82.

Huntenburg JM, Bazin P-L, Goulas A, Tardif CL, Villringer A, Margulies DS (2017) A systematic relationship between functional connectivity and intracortical myelin in the human cerebral cortex. Cereb Cortex 27:981997.

Ito T, Bai J, Ostry DJ (2020) Contribution of sensory memory to speech motor learning. J Neurophysiol 124:1103-1109. 
Jenkinson M, Beckmann CF, Behrens TEJ, Woolrich MW, Smith SM (2012) FSL. Neuroimage 62:782-790.

Jo HJ, Saad ZS, Simmons WK, Milbury LA, Cox RW (2010) Mapping sources of correlation in resting state FMRI, with artifact detection and removal. Neuroimage 52:571-582.

Johnson JF, Belyk M, Schwartze M, Pinheiro AP, Kotz SA (2019) The role of the cerebellum in adaptation: ALE meta-analyses on sensory feedback error. Hum Brain Mapp 40:3966-3981.

Kellogg WN (1968) Communication and language in the home-raised chimpanzee. Science 162:423-427.

Kelly RE, Jr, Alexopoulos GS, Wang Z, Gunning FM, Murphy CF, Morimoto SS, Kanellopoulos D, Jia Z, Lim KO, Hoptman MJ (2010) Visual inspection of independent components: defining a procedure for artifact removal from fMRI data. J Neurosci Methods 189:233-245.

Kennerley SW, Sakai K, Rushworth MFS (2004) Organization of action sequences and the role of the pre-SMA. J Neurophysiol 91:978-993.

Kim KS, Daliri A, Flanagan JR, Max L (2020) Dissociated development of speech and limb sensorimotor learning in stuttering: speech auditorymotor learning is impaired in both children and adults who stutter. Neuroscience 451:1-21.

Kleber B, Zeitouni AG, Friberg A, Zatorre RJ (2013) Experience-dependent modulation of feedback integration during singing: role of the right anterior insula. J Neurosci 33:6070-6080.

Kleber B, Friberg A, Zeitouni A, Zatorre R (2017) Experience-dependent modulation of right anterior insula and sensorimotor regions as a function of noise-masked auditory feedback in singers and nonsingers. Neuroimage 147:97-110.

Kostopoulos P, Petrides M (2003) The mid-ventrolateral prefrontal cortex: insights into its role in memory retrieval. Eur J Neurosci 17:1489-1497.

Kostopoulos P, Petrides M (2016) Selective memory retrieval of auditory what and auditory where involves the ventrolateral prefrontal cortex. Proc Natl Acad Sci U S A 113:1919-1924.

Kostopoulos P, Albanese M-C, Petrides M (2007) Ventrolateral prefrontal cortex and tactile memory disambiguation in the human brain. Proc Natl Acad Sci U S A 104:10223-10228.

Krishnan S, Leech R, Mercure E, Lloyd-Fox S, Dick F (2015) Convergent and divergent $\mathrm{fMRI}$ responses in children and adults to increasing language production demands. Cereb Cortex 25:3261-3277.

Lametti DR, Nasir SM, Ostry DJ (2012) Sensory preference in speech production revealed by simultaneous alteration of auditory and somatosensory feedback. J Neurosci 32:9351-9358.

Lima CF, Krishnan S, Scott SK (2016) Roles of supplementary motor areas in auditory processing and auditory imagery. Trends Neurosci 39:527-542.

Loh KK, Procyk E, Neveu R, Lamberton F, Hopkins WD, Petrides M, Amiez C (2020) Cognitive control of orofacial motor and vocal responses in the ventrolateral and dorsomedial human frontal cortex. Proc Natl Acad Sci U S A 117:4994-5005.

Lutkenhoff ES, Rosenberg M, Chiang J, Zhang K, Pickard JD, Owen AM, Monti MM (2014) Optimized brain extraction for pathological brains (optiBET). PLoS One 9:e115551.

MacDonald EN, Johnson EK, Forsythe J, Plante P, Munhall KG (2012) Children's development of self-regulation in speech production. Curr Biol 22:113-117.

Margulies DS, Petrides M (2013) Distinct parietal and temporal connectivity profiles of ventrolateral frontal areas involved in language production. J Neurosci 33:16846-16852.

Margulies DS, Ghosh SS, Goulas A, Falkiewicz M, Huntenburg JM, Langs G, Bezgin G, Eickhoff SB, Castellanos FX, Petrides M, Jefferies E, Smallwood J (2016) Situating the default-mode network along a principal gradient of macroscale cortical organization. Proc Natl Acad Sci U S A 113:1257412579.

McLaren DG, Ries ML, Xu G, Johnson SC (2012) A generalized form of context-dependent psychophysiological interactions (gPPI): a comparison to standard approaches. Neuroimage 61:1277-1286.

Morecraft RJ, Tanji J (2009) Cingulofrontal interactions and the cingulate motor areas. In: Cingulate neurobiology and disease (Vogt BA, ed), pp 113-144. Oxford: Oxford University UP.

Munhall KG, MacDonald EN, Byrne SK, Johnsrude I (2009) Talkers alter vowel production in response to real-time formant perturbation even when instructed not to compensate. J Acoust Soc Am 125:384-390.

Nasir SM, Ostry DJ (2008) Speech motor learning in profoundly deaf adults. Nat Neurosci 11:1217-1222.
Niziolek CA, Guenther FH (2013) Vowel category boundaries enhance cortical and behavioral responses to speech feedback alterations. J Neurosci 33:12090-12098.

Paquola C, Vos De Wael R, Wagstyl K, Bethlehem RAI, Hong S-J, Seidlitz J, Bullmore ET, Evans AC, Misic B, Margulies DS, Smallwood J, Bernhardt BC (2019) Microstructural and functional gradients are increasingly dissociated in transmodal cortices. PLoS Biol 17:e3000284.

Parrell B, Agnew Z, Nagarajan S, Houde J, Ivry RB (2017) Impaired Feedforward Control and Enhanced Feedback Control of Speech in Patients with Cerebellar Degeneration. J Neurosci 37:9249-9258.

Petrides M (2014) Neuroanatomy of language regions of the human brain. San Diego: Academic Press.

Picard N, Strick PL (1996) Motor areas of the medial wall: a review of their location and functional activation. Cereb Cortex 6:342-353.

Picard N, Strick PL (2001) Imaging the premotor areas. Curr Opin Neurobiol 11:663-672.

Rauschecker JP, Scott SK (2009) Maps and streams in the auditory cortex: nonhuman primates illuminate human speech processing. Nat Neurosci 12:718-724.

Rochet-Capellan A, Ostry DJ (2011) Simultaneous acquisition of multiple auditory-motor transformations in speech. J Neurosci 31:2657-2662.

Rogalsky C, Poppa T, Chen K-H, Anderson SW, Damasio H, Love T, Hickok G (2015) Speech repetition as a window on the neurobiology of auditorymotor integration for speech: A voxel-based lesion symptom mapping study. Neuropsychologia 71:18-27.

Rossi C, Chau CW, Leech KA, Statton MA, Gonzalez AJ, Bastian AJ (2019) The capacity to learn new motor and perceptual calibrations develops concurrently in childhood. Sci Rep 9:9322.

Rummel C, Verma RK, Schöpf V, Abela E, Hauf M, Berruecos JFZ, Wiest R (2013) Time course based artifact identification for independent components of resting-state FMRI. Front Hum Neurosci 7:214.

Saad ZS, Reynolds RC, Argall B, Japee S, Cox RW (2005) SUMA: An interface for surface-based intra- and inter-subject analysis with AFNI. In: 2004 2nd IEEE International Symposium on Biomedical Imaging: Macro to Nano (IEEE Cat No. 04EX821). IEEE.

Seghier ML, Hope TMH, Prejawa S, Parker Jones ' $\mathrm{O}^{\circ}$, Vitkovitch M, Price CJ (2015) A trade-off between somatosensory and auditory related brain activity during object naming but not reading. J Neurosci 35:4751-4759.

Sherwood CC, Gómez-Robles A (2017) Brain Plasticity and Human Evolution. Annu Rev Anthropol 46:399-419.

Shiller DM, Rochon M-L (2014) Auditory-perceptual learning improves speech motor adaptation in children. J Exp Psychol Hum Percept Perform 40:1308-1315.

Shiller DM, Gracco VL, Rvachew S (2010) Auditory-motor learning during speech production in 9-11-year-old children. PLoS One 5:e12975.

Shum M, Shiller DM, Baum SR, Gracco VL (2011) Sensorimotor integration for speech motor learning involves the inferior parietal cortex. Eur J Neurosci 34:1817-1822.

Sidarta A, van Vugt FT, Ostry DJ (2018) Somatosensory working memory in human reinforcement-based motor learning. J Neurophysiol 120:32753286.

Smith MA, Ghazizadeh A, Shadmehr R (2006) Interacting adaptive processes with different timescales underlie short-term motor learning. PLoS Biol 4:e179.

Smith SM, Fox PT, Miller KL, Glahn DC, Fox PM, Mackay CE, Filippini N, Watkins KE, Toro R, Laird AR, Beckmann CF (2009) Correspondence of the brain's functional architecture during activation and rest. Proc Natl Acad Sci U S A 106:13040-13045.

Smith SM, Beckmann CF, Andersson J, Auerbach EJ, Bijsterbosch J, Douaud G, Duff E, Feinberg DA, Griffanti L, Harms MP, Kelly M, Laumann T, Miller KL, Moeller S, Petersen S, Power J, Salimi-Khorshidi G, Snyder AZ, Vu AT, Woolrich MW, et al. (2013) Resting-state fMRI in the human connectome project. Neuroimage 80:144-168.

Sotiras A, Toledo JB, Gur RE, Gur RC, Satterthwaite TD, Davatzikos C (2017) Patterns of coordinated cortical remodeling during adolescence and their associations with functional specialization and evolutionary expansion. Proc Natl Acad Sci U S A 114:3527-3532.

Tourville JA, Guenther FH (2011) The DIVA model: A neural theory of speech acquisition and production. Lang Cogn Process 26:952-981.

Tourville JA, Reilly KJ, Guenther FH (2008) Neural mechanisms underlying auditory feedback control of speech. Neuroimage 39:1429-1443. 
Tremblay S, Shiller DM, Ostry DJ (2003) Somatosensory basis of speech production. Nature 423:866-869.

Uğurbil K, Xu J, Auerbach EJ, Moeller S, Vu AT, Duarte-Carvajalino JM, Lenglet C, Wu X, Schmitter S, Van de Moortele PF, Strupp J, Sapiro G, De Martino F, Wang D, Harel N, Garwood M, Chen L, Feinberg DA, Smith SM, Miller KL, et al. (2013) Pushing spatial and temporal resolution for functional and diffusion MRI in the Human Connectome Project. Neuroimage 80:80-104.

van Brenk F, Terband H (2020) Compensatory and adaptive responses to realtime formant shifts in adults and children. J Acoust Soc Am 147:2261.

Van Essen DC, Ugurbil K, Auerbach E, Barch D, Behrens TEJ, Bucholz R, Chang A, Chen L, Corbetta M, Curtiss SW, Della Penna S, Feinberg D, Glasser MF, Harel N, Heath AC, Larson-Prior L, Marcus D, Michalareas G, Moeller S, Oostenveld R, et al. (2012) The Human Connectome Project: a data acquisition perspective. Neuroimage 62:2222-2231.

Váša F, Romero-Garcia R, Kitzbichler MG, Seidlitz J, Whitaker KJ, Vaghi MM, Kundu P, Patel AX, Fonagy P, Dolan RJ, Jones PB, Goodyer IM, Vértes, PE, Bullmore ET. (2020) Conservative and disruptive modes of adolescent change in human brain functional connectivity. Proc Natl Acad Sci U S A 117:3248-3253.
Vasudevan EVL, Torres-Oviedo G, Morton SM, Yang JF, Bastian AJ (2011) Younger is not always better: development of locomotor adaptation from childhood to adulthood. J Neurosci 31:3055-3065.

Vázquez-Rodríguez B, Suárez LE, Markello RD, Shafiei G, Paquola C, Hagmann P, van den Heuvel MP, Bernhardt BC, Spreng RN, Misic B (2019) Gradients of structure-function tethering across neocortex. Proc Natl Acad Sci U S A 116:21219-21227.

Vergara J, Rivera N, Rossi-Pool R, Romo R (2016) A neural parametric code for storing information of more than one sensory modality in working memory. Neuron 89:54-62.

von Economo CF, Koskinas GN (1925) Die cytoarchitektonik der hirnrinde des erwachsenen menschen. Berlin: J. Springer.

Woolnough O, Forseth KJ, Rollo PS, Tandon N (2019) Uncovering the functional anatomy of the human insula during speech. eLife 8:e53086.

Yates AJ (1965) Delayed auditory feedback and shadowing. Q J Exp Psychol 17:125-131.

Zarate JM, Zatorre RJ (2008) Experience-dependent neural substrates involved in vocal pitch regulation during singing. Neuroimage 40:1871-1887.

Zheng ZZ, Vicente-Grabovetsky A, MacDonald EN, Munhall KG, Cusack R, Johnsrude IS (2013) Multivoxel patterns reveal functionally differentiated networks underlying auditory feedback processing of speech. J Neurosci 33:4339-4348 